\title{
Stent versus Balloon Dilation for the Treatment of Dominant Strictures in Primary Sclerosing Cholangitis: A Systematic Review and Meta-Analysis
}

\author{
Marina Tucci Gammaro Baldavira Ferreira', Igor Braga Ribeiro', Diogo Turiani Hourneaux de Moura', Thomas R. McCarty ${ }^{2}$, \\ Alberto Machado da Ponte Neto', Galileu Ferreira Ayala Farias', Antônio Afonso de Miranda Neto', Pedro Victor Aniz Gomes de \\ Oliveira', Wanderley Marques Bernardo ${ }^{1}$ and Eduardo Guimarães Hourneaux de Moura ${ }^{1}$ \\ ${ }^{1}$ Gastrointestinal Endoscopy Unity, Hospital das Clínicas da Faculdade de Medicina da Universidade de São Paulo - HC/FMUSP, São \\ Paulo, SP, Brazil, 'Division of Gasteoenterology, Hepatology and Endoscopy, Brigham and Women's Hospital, Harvard Medical School, \\ Boston, MA, USA
}

Background/Aims: The endoscopic management of primary sclerosing cholangitis (PSC)-associated dominant strictures remains challenging. This systematic review and meta-analysis aimed to compare balloon dilation and stent placement in the treatment of dominant strictures among PSC patients.

Methods: Literature searches on MEDLINE, EMBASE, Cochrane CENTRAL and Lilacs/Bireme were performed for studies published until December 2020. Measured outcomes included clinical efficacy, stricture recurrence, cumulative recurrencefree rate, transplant rate, 5-year survival rate, and adverse events (i.e., pancreatitis, cholangitis, bleeding, perforation and death).

Results: A total of 5 studies $(n=467)$ were included. Based on pooled analyses, there were no differences in clinical efficacy (risk difference [RD], $-0.13 ; 95 \%$ confidence interval $[\mathrm{CI}],-0.58$ to $0.33 ; \mathrm{I}^{2}=93 \%$ ) or transplant rates $(\mathrm{RD},-0.09 ; 95 \% \mathrm{CI},-0.19$ to 0.01 ; $\mathrm{I}^{2}=0 \%$ ); however, the risk of occurrence of adverse events was lower with balloon dilatation than with stent placement (RD, $-0.34 ; 95 \% \mathrm{CI},-0.45$ to $\left.-0.23 ; \mathrm{I}^{2}=61 \%\right)$. Among the types of adverse events reported, only the rates of cholangitis/bacteremia were significantly lower in balloon dilation patients (RD, $-0.19 ; 95 \% \mathrm{CI},-0.25$ to $-0.13 ; \mathrm{I}^{2}=51 \%$ ).

Conclusions: Compared to balloon dilation, stent placement for dominant strictures in PSC appeared to have higher complication rates without significant differences in efficacy. Clin Endosc 2021;54:833-842

Key Words: Balloon dilation; Dominant stricture; Primary sclerosing cholangitis; Stent

\section{INTRODUCTION}

Primary sclerosing cholangitis (PSC) is a chronic cholestatic disease of the hepatobiliary system, characterized by pro-

Received: January 17, 2021 Revised: January 30, 2021

Accepted: February 2, 2021

Correspondence: Igor Braga Ribeiro

Gastrointestinal Endoscopy Unity, Hospital das Clínicas da Faculdade de Medicina da Universidade de São Paulo, Rua, Av. Dr. Enéas Carvalho de Aguiar, 255 Cerqueira César, São Paulo, SP 05403-010, Brazil

Tel: +55-929-8137-7788, Fax: +55-11-2661-6467, E-mail: igorbraga1@gmail.com ORCID: https://orcid.org/0000-0003-1844-8973

(c) This is an Open Access article distributed under the terms of the Creative Commons Attribution Non-Commercial License (http://creativecommons.org/ licenses/by-nc/3.0) which permits unrestricted non-commercial use, distribution, and reproduction in any medium, provided the original work is properly cited. gressive inflammation, fibrosis, and stricturing of the intraand extra-hepatic bile ducts. ${ }^{1-3}$ Histopathologically, PSC is characterized by inflammation and fibrosis of the intra- and extra-hepatic ductal epithelium, which can ultimately progress to end-stage liver disease and cirrhosis, and increase the risk for cholangiocarcinoma. ${ }^{4,5}$ The presentation of PSC is highly variable, ranging from asymptomatic in the early stages, to recurrent episodes of cholangitis with fever, jaundice, pruritus, abdominal pain and fatigue. However, median survival tends to be significantly decreased in patients who are symptomatic at the time of diagnosis. ${ }^{6,7}$ Epidemiological studies of PSC have reported a prevalence rate of approximately 1 per 10,000, and incidence rates between 0.4 and 2.0 per 100,000 per year in Northern Europe and the US. ${ }^{8}$ PSC conforms to the definition of rare diseases, affecting less than 200,000 individuals in the 
US and less than 5 per 10,000 inhabitants in the EU. The typical PSC patient is, however, a 30-40-year-old male (a young person of working age). The increased risk of biliary and colorectal cancer in PSC has been firmly established, and has been considered of major clinical importance. In a multi-center study of 7,119 PSC patients, hepatobiliary malignancy was diagnosed in $10.9 \%{ }^{9}$

Given the progressive nature of the disease and a median survival after diagnosis of 10-12 years without liver transplantation, early diagnosis with magnetic resonance cholangiography or endoscopic retrograde endoscopic cholangiography (ERCP) remains key, ${ }^{6,10-13}$ mainly due to their proven safety. ${ }^{14-18}$ Most guidelines, including those of the European Association for the Study of the Liver /European Society of Gastrointestinal Endoscopy ${ }^{19}$ and of the British Society of Gastroenterology, ${ }^{20}$ recommend ERCP for diagnosis only if therapeutic interventions or biopsies are required during the procedure, due to its invasive nature. Although liver transplantation remains the treatment of choice for advanced diseases and represents the only curative therapy for PSC, patients with dominant extra-hepatic biliary strictures may potentially benefit from endoscopic management. ${ }^{7,11,21}$ Dominant biliary strictures are functionally defined as a narrowing within the biliary tree that halts the normal flow of bile and, by most consensus guidelines, is considered a stricture $<1.5 \mathrm{~mm}$ in the bile duct or $<1 \mathrm{~mm}$ in the hepatic ducts, with distance $<2 \mathrm{~cm}$ from the bifurcation in the hepatic hilum. ${ }^{22,23}$ During the natural history of the disease, many patients experience symptoms such as pruritus, right upper quadrant pain, fatigue, fever, and jaundice due to impeded biliary drainage. In approximately $60 \%$ of cases, dominant strictures (DS), which may be superimposed on diffuse ductal diseases, are the principal cause of such complaints. The annual incidence of DS has been estimated to be $8-10 \%{ }^{24}$ Dominant strictures may also exacerbate cholestatic symptoms, including worsening pruritus or the development of cholangitis, and may hence benefit from endoscopic therapy with either balloon dilation or stent placement. These endoscopic procedures aim to improve biliary tree patency, prevent or reduce cholestasis, relieve symptoms or pruritus, improve patient quality of life, and potentially increase patient survival. ${ }^{11,25-27}$

Endoscopic treatment for dominant strictures typically involves either balloon dilation or placement of a temporary stent through ERCP. ${ }^{23,28}$ Recent studies have shown that balloon dilation may be sufficient to maintain patency without the need for stent placement, ${ }^{29}$ allowing for clinical improvement in patients, while avoiding surgical procedures prior to liver transplantation. ${ }^{24}$ However, early restenosis has been portrayed as a potential disadvantage of balloon dilation, with the need for repeated interventions with stent placement or a combination of techniques. ${ }^{24}$ Although stent placement may be more effective for some dominant strictures, the ideal duration of stent placement remains unknown, and the additional procedure required for stent removal carries a risk of stent occlusion, with possible cholangitis and sepsis. ${ }^{24,30}$ The British Society of Gastroenterology and UK-PSC guidelines for the diagnosis and management of PSC recommend biliary dilation, rather than biliary stent insertion, as the management of choice. However, this recommendation was based on a moderate quality of evidence. ${ }^{20}$ Given the current clinical conundrum, the decision to perform balloon dilation or stent placement is largely driven by procedural familiarity. Therefore, the primary aim of this systematic review and meta-analysis was to determine the optimal endoscopic treatment approach (i.e., balloon dilation or stent placement) for dominant biliary strictures in PSC.

\section{METHODS}

\section{Protocol and registration}

This systematic review was carried out in accordance with the Cochrane Handbook for Systematic Reviews of Interventions and the Preferred Reporting Items for Systematic Reviews and Meta-Analysis (PRISMA) guideline. ${ }^{31}$ The study was registered by the International Prospective Register of Systematic Reviews (PROSPERO; registration number, CRD42019137645).

\section{Eligibility criteria}

All comparative, retrospective and prospective observational studies, as well as randomized controlled trials, that involved patients with PSC were included in this analysis. Inclusion criteria for the patients were a diagnosis of at least one dominant stricture in the intra- or extra-hepatic bile ducts, and a history of biliary stent placement (endoscopic or percutaneous) or balloon dilation. There were no restrictions on the language or year of publication of the studies. Studies that did not provide sufficient data for analysis of outcomes were excluded.

\section{Information sources}

Two independent researchers (MTGBF and IBR) extracted the data according to a predefined data extraction form. Disagreements were resolved by consultation with a third researcher (EGHM).

\section{Literature search}

The article search was carried out from May 2019 to December 2020, in electronic databases including PubMed/ MEDLINE, EMBASE, Cochrane Library and LILACS. There 
were no restrictions regarding the language or year of publication. The search strategy used was as follows: (cholangitis, sclerosing OR sclerosing cholangitides OR sclerosing cholangitis) AND (prostheses and implants OR prosthetic implants OR endoprosthesis OR stent* OR dilatation OR dilation OR balloon).

\section{Study selection}

During the search, studies compatible with the theme were identified by title, and a new assessment of the eligibility criteria was performed through the abstracts, followed by the complete text to better suit the study objectives (Fig. 1).

\section{Measured outcomes}

The analyzed outcomes included clinical efficacy, recurrence, cumulative recurrence-free rate, transplant rate, 5-year survival rate, mortality, total adverse events (including cholangitis, pancreatitis, perforation, and bleeding), progression to cholangiocarcinoma, pain, ascites, as well as the clinical in- dications for each procedure (jaundice, recurrent cholangitis, abdominal pain, pruritus, and fever).

\section{Risk of bias}

The risk of bias in each study was assessed using the Revised Cochrane Risk-of-Bias Tool (RoB-2) ${ }^{32}$ randomized clinical trials, and the Revised Cochrane Risk-of-Bias Tool to Assess Non-randomized Studies of Interventions (ROBINS-I) ${ }^{33}$ for retrospective studies. The analysis was performed separately for each outcome and for each corresponding study.

The quality of evidence for each of the pre-specified outcomes was assessed according to the objective criteria of the Grading of Recommendations Assessment, Development and Evaluation (GRADE) system ${ }^{34}$, by using GRADEpro (Guideline Development Tool Software).

\section{Assessment of Heterogeneity}

The value of heterogeneity (inconsistency) was assessed according to the Chi-square $\left(\chi^{2}\right)$ and Higgins method $\left(I^{2}\right)$. Heterogeneity values greater than $50 \%$ were considered high,
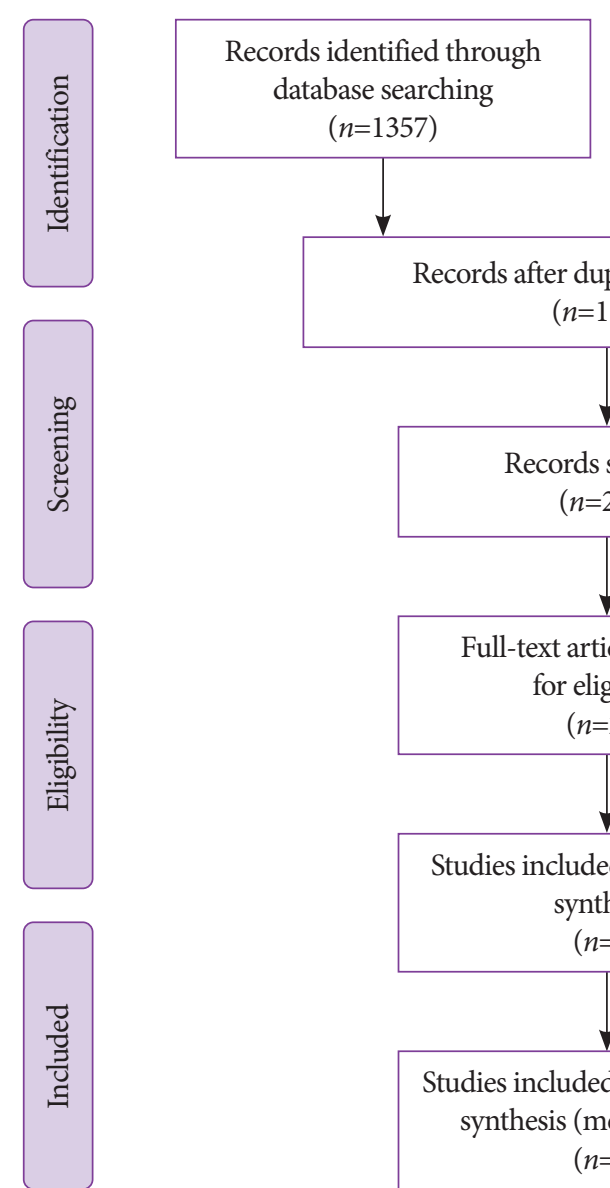

Additional records identified through other sources

$$
(n=0)
$$

Records excluded $(n=210)$

Full-text articles excluded, with reasons ${ }^{\star}$ $(n=15)$

Fig. 1. Flow chart of study selection. *: Narrative reviews (9), guidelines (2) and case reports (4) 
and the random-effect model would be applied for analysis. For heterogeneity values less than $50 \%$, the fixed-effect model would be used.

\section{Statistical analyses}

For dichotomous variables, risk difference (RD) was calculated using the Mantel-Haenszel method, with a 95\% confidence interval (CI). For continuous variables, mean value of difference was with the inverse variance method and a 95\% CI. Absolute number, mean, and standard deviation were used for data analysis. For studies that did not include means and standard deviations, the data were standardized using mathematical formulas ${ }^{35}$. The RevMan 5 software (Review Manager version 5.3.5; Cochrane Collaboration, Oxford, UK) was used for data analysis, as well as to generate the forest plot graphs and to calculate the confidence intervals.

\section{RESULTS}

\section{Study selection and search results}

Initially, a total of 1,118 studies were identified through the search strategies as mentioned above. After evaluating the titles, 230 studies with a theme pertinent to that proposed by this study were identified. After subsequent screening of the abstracts, 20 were selected as possible candidates, and a full-text review was performed. Eventually, a total of five articles $^{24,29,36-38}$ were eligible for qualitative and quantitative review and meta-analysis. A complete flow diagram of the study selection process is highlighted in Fig. 1.

\section{Study characteristics}

Five studies were included in this systematic review and meta-analysis. Of these, only one was a randomized clinical trial $^{24}$, while the others were retrospective cohort studies. ${ }^{29,36-38}$ The total number of patients analyzed was 467, of which 277 underwent stent placement and 190 underwent balloon dilatation of dominant strictures (Table 1).

The different techniques applied in each study included balloon dilation before stent placement, ${ }^{29,36,38}$ percutaneous transhepatic stent placement, as well as short- and long-term endoscopic stents. ${ }^{37}$ In the study by Ponsioen et al., ${ }^{24}$ balloon dilation with or without the use of Soehendra dilators was allowed prior to stent placement, with indication determined by the physician at the time of examination. The definition of short- and long-term stents also varied according to studies. Al-Lehibi et al. ${ }^{37}$ defined short-term stents as those placed for $<4$ weeks, while Navaneethan et $\mathrm{al}^{36}$ considered a duration of $<7$ days. While dilation and stent placement in the studies by Al-Lehibi et al., ${ }^{37}$ Han et al., ${ }^{38}$ Navaneethan et al., ${ }^{36}$ and

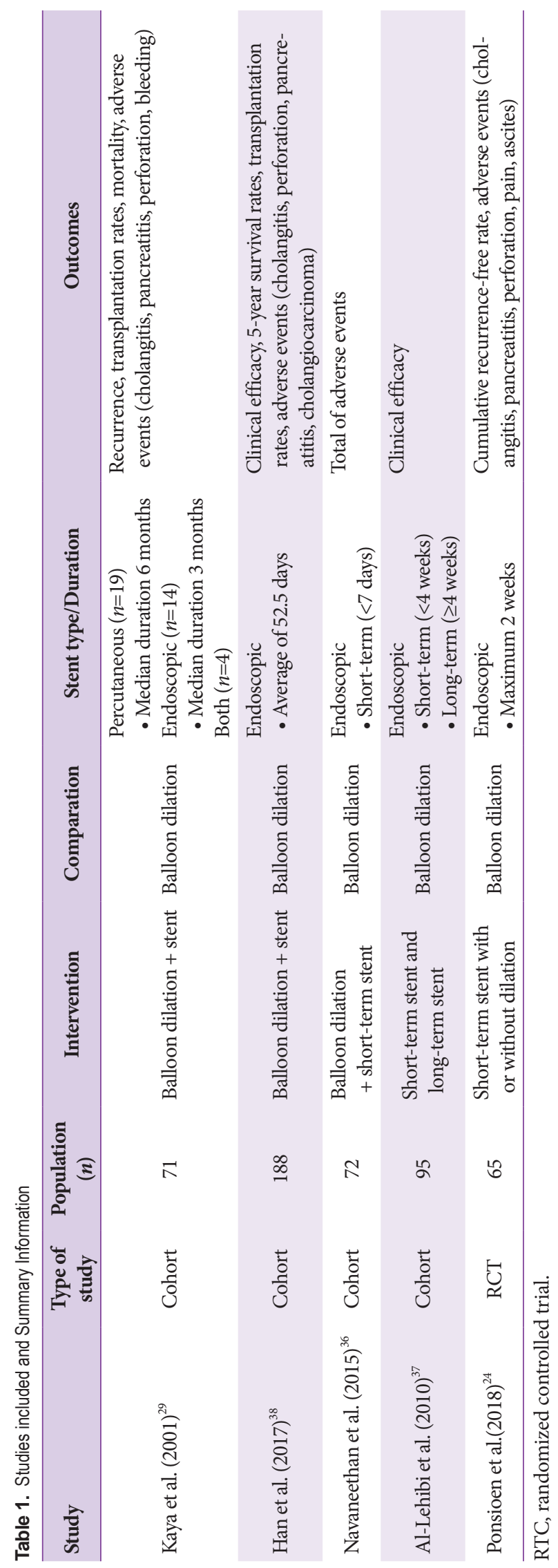


Ponsioen et $\mathrm{al}^{24}$ were performed via ERCP, those in the study by Kaya et al. ${ }^{29}$ were carried out by ERCP, percutaneously, or with both techniques combined. Percutaneous transhepatic interventions were performed when adequate biliary cannulation was not possible, when failure to traverse the stricture with a guidewire occurred, or when the stricture hindered the passage of a dilator during an endoscopic procedure. In these cases, internal biliary drainage was performed at 6-8-week intervals while catheter access was maintained, or when cholangitis or jaundice recurred. ${ }^{29}$ Stents placed by ERCP, on the other hand, were routinely removed or changed either after 3-4 months, or when cholangitis or jaundice recurred. It should be noted, however, that the percutaneous treatment for dominant strictures has its own intrinsic complications, such as the increased risk of bleeding or bile duct perforation. ${ }^{29}$

\section{Primary and secondary outcomes}

Two studies, ${ }^{37,38}$ including a total of 259 patients, documented the clinical efficacy of stent placement versus balloon dilation of dominant strictures. Pooled analyses of these studies revealed no significant differences between balloon dilation and stent placement (RD, -0.13 ; $95 \% \mathrm{CI},-0.58$ to 0.33 ; $I^{2}=93 \%$ ) (Fig. 2A). With regards to stricture recurrence, only one study ${ }^{29}$ reported this outcome, with similar but higher rates reported in the dilation group. Due to this, a meta-anal- ysis on such data was not possible. Two studies ${ }^{29,38}$ compared the transplant rates in a total of 259 patients, but reported no significant differences in the analyzed outcome $(\mathrm{RD},-0.09$; $95 \%$ CI, -0.19 to $0.01 ; I^{2}=0 \%$ ) (Fig. 2B). Only one study ${ }^{38}$ compared the 5-year survival rate, and reported higher rates in the dilation group. Mortality ${ }^{29}$ as a result of balloon dilation or stent placement was also reported in only one study, and demonstrated similar results.

A total of three studies ${ }^{24,29,36}$ reported information on the total number of adverse events. Among the 206 patients included, balloon dilation associated with a significantly decreased rate of total adverse events (RD, $-0.34 ; 95 \% \mathrm{CI},-0.45$ to $-0.23 ; I^{2}=61 \%$ ) (Fig. $3 \mathrm{~A}$ ). When stratified by the type of adverse event, only the rates of cholangitis/bacteremia were significantly different, with four studies ${ }^{24,29,36,38}$ on 394 patients reporting a significantly lower rate among balloon dilation patients (RD, -0.19 ; 95\% CI, -0.25 to $-0.13 ; I^{2}=51 \%$ ) (Fig. $3 \mathrm{~B}$ ). No significant differences were reported in the development of post-procedure pancreatitis $(\mathrm{RD},-0.08$; $95 \% \mathrm{CI},-0.19$ to 0.03 ; $I^{2}=65 \%$ ), perforation (RD, $-0.0195 \% \mathrm{CI}-0.05$ to $0.03 ; I^{2}=1 \%$ ), or cholangiocarcinoma (RD, 0.00 ; $95 \% \mathrm{CI},-0.08$ to 0.07 ; $I^{2}=0 \%$ ) (Fig. 3C-E). Bleeding ${ }^{29}$ and pain ${ }^{24}$ were complications that each appeared in only one study, rendering it impossible to meta-analyze such data. The rates of both complications, however, were higher in the stent group, thereby favoring the

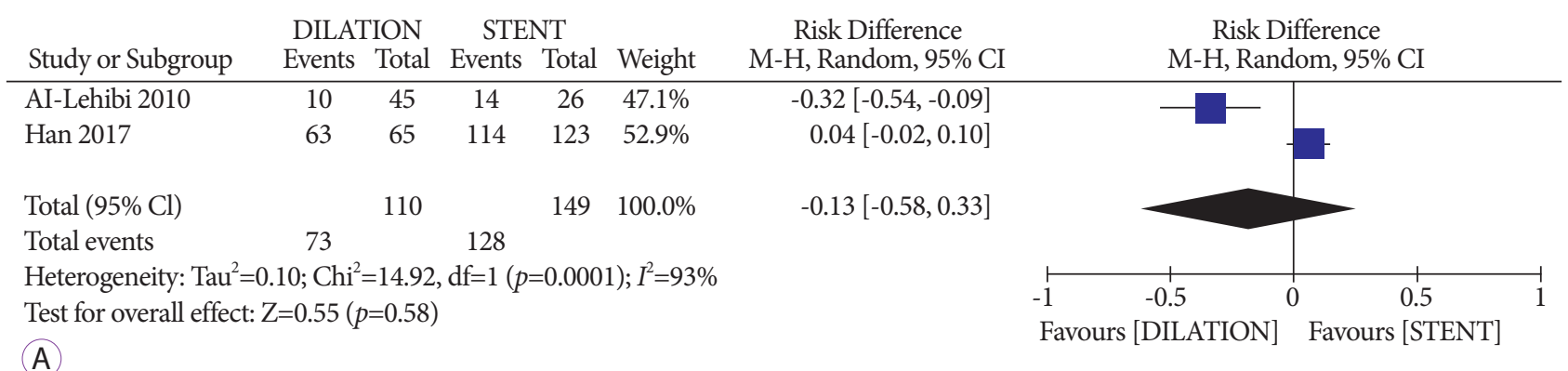

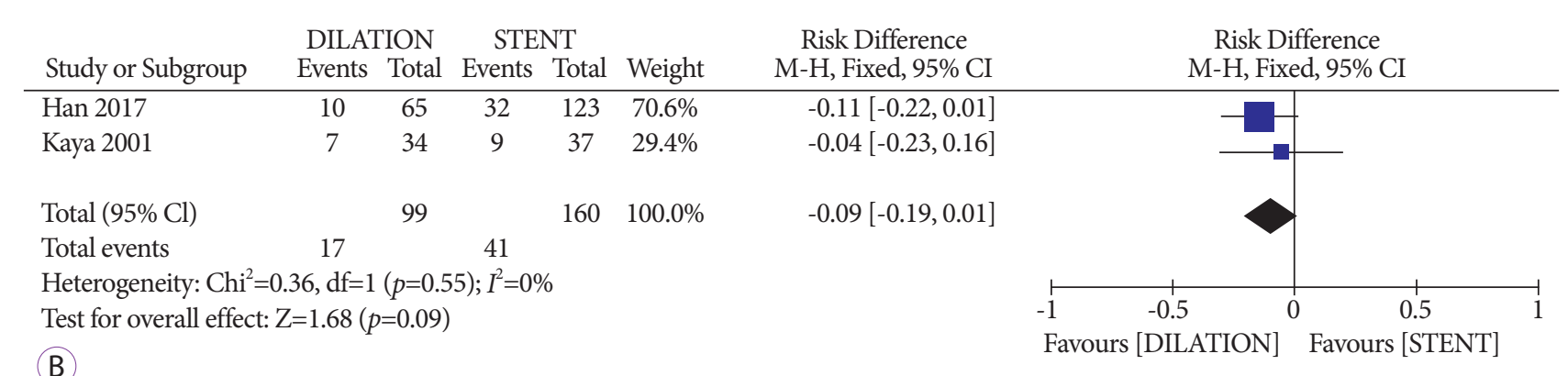

Fig. 2. Stent $\times$ ballon dilation. (A) Forest plot of clinical efficacy. (B) Forest plot of transplant rate. $\mathrm{Cl}$, confidence interval. 


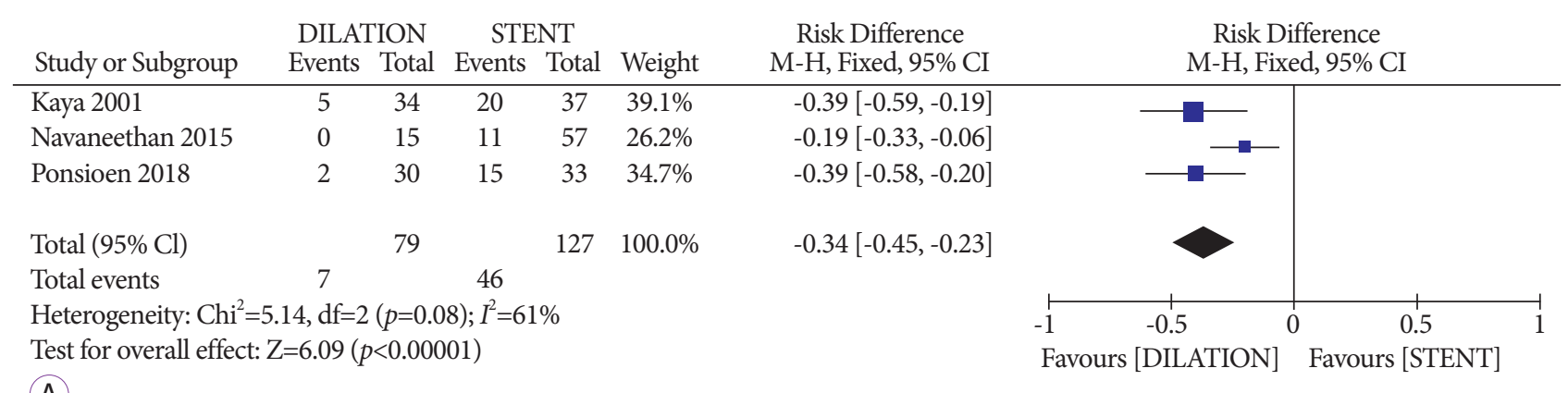

(A)

\begin{tabular}{|c|c|c|c|c|c|c|}
\hline \multirow[b]{2}{*}{ Study or Subgroup } & \multicolumn{2}{|c|}{ DILATION } & \multicolumn{2}{|c|}{ STENT } & \multirow[b]{2}{*}{ Weight } & \multirow{2}{*}{$\begin{array}{c}\text { Risk Difference } \\
\text { M-H, Fixed, 95\% CI }\end{array}$} \\
\hline & Events & Total & Events & Total & & \\
\hline Han 2017 & 1 & 65 & 27 & 123 & $48.4 \%$ & $-0.20[-0.28,-0.13]$ \\
\hline Kaya 2001 & 1 & 34 & 12 & 37 & $20.2 \%$ & $-0.29[-0.46,-0.13]$ \\
\hline Navaneethan 2015 & 0 & 15 & 6 & 57 & $13.5 \%$ & $-0.11[-0.22,0.01]$ \\
\hline Ponsioen 2018 & 1 & 30 & 4 & 33 & $17.9 \%$ & $-0.09[-0.22,0.04]$ \\
\hline Total $(95 \% \mathrm{Cl})$ & & 144 & & 250 & $100.0 \%$ & $-0.19[-0.25,-0.13]$ \\
\hline Total events & 3 & & 49 & & & \\
\hline
\end{tabular}

Heterogeneity: $\mathrm{Chi}^{2}=6.09, \mathrm{df}=3(p=0.11) ; I^{2}=51 \%$

Test for overall effect: $\mathrm{Z}=6.39(p<0.00001)$

(B)

DILATION STENT

Risk Difference

\begin{tabular}{lccccc} 
Study or Subgroup & Events & Total & Events & Total & Weight \\
\hline Han 2017 & 7 & 65 & 12 & 123 & $38.4 \%$
\end{tabular}

M-H, Random, 95\% CI

Risk Difference M-H, Fixed, 95\% CI

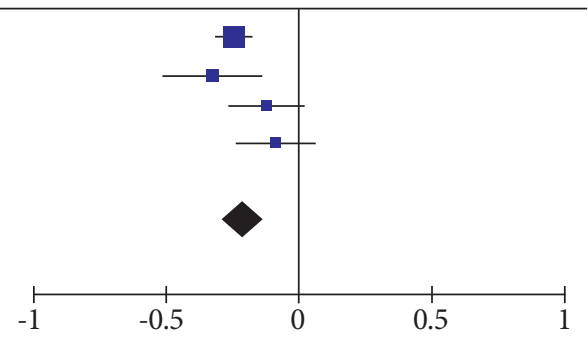

Favours [DILATION] Favours [STENT]

$\begin{array}{llllll}\text { Kaya } 2001 & 0 & 34 & 3 & 37 & 36.6 \%\end{array}$

Ponsioen 2018

$\begin{array}{lllll}1 & 30 & 8 & 33 & 25.1 \%\end{array}$

$0.01[-0.08,0.10]$

$-0.08[-0.18,0.02]$

$-0.21[-0.37,-0.05]$

Total $(95 \% \mathrm{Cl})$

129

$193 \quad 100.0 \%$

Total events

8 23

Heterogeneity: $\mathrm{Tau}^{2}=0.01 ; \mathrm{Chi}^{2}=5.76, \mathrm{df}=2(p=0.06) ; I^{2}=65 \%$

Test for overall effect: $Z=1.38(p=0.17)$

(C)

\begin{tabular}{lccccc} 
Study or Subgroup & \multicolumn{2}{c}{ DILATION } & \multicolumn{2}{c}{ STENT } & \\
Events & Total & Events & Total & Weight \\
\hline Han 2017 & 2 & 65 & 2 & 123 & $56.0 \%$
\end{tabular}

$\begin{array}{llllll}\text { Kaya } 2001 & 2 & 34 & 5 & 37 & 23.3 \%\end{array}$

Ponsioen 2018

$\begin{array}{lllll}0 & 30 & 0 & 33 & 20.7 \%\end{array}$

$-0.08[-0.19,0.03]$

Total $(95 \% \mathrm{Cl})$

129

$193 \quad 100.0 \%$

Total events

4

7

Heterogeneity: $\mathrm{Chi}^{2}=2.02, \mathrm{df}=2(p=0.36) ; I^{2}=1 \%$

Test for overall effect: $Z=0.44(p=0.66)$

(D)

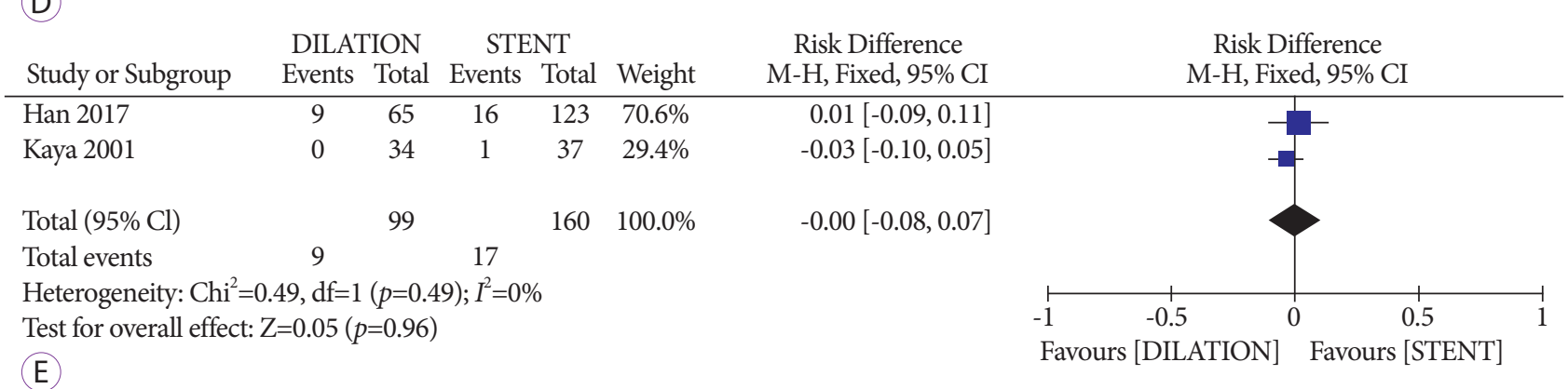

Fig. 3. Stent $\times$ ballon dilation adverse events. (A) Forest plot of total adverse events. (B) Forest plot of cholangitis/bacteremia. (C) Forest plot of pancreatitis. (D) Forest plot of perforation. $(\mathrm{E})$ Forest plot of cholangiocarcinoma. $\mathrm{Cl}$, confidence interval. 
balloon dilation approach.

With regards to the clinical indications for each procedure, recurrent cholangitis was more common in the balloon dilation cohort as compared to the stent placement group (RD, -0.20 ; $95 \% \mathrm{CI},-0.28$ to $\left.-0.13 ; I^{2}=2 \%\right)^{29,38}$. No statistical differences in jaundice (RD, $-0.07 ; 95 \% \mathrm{CI},-0.20$ to $0.05 ; I^{2}=35 \%$ ), abdominal pain (RD, $-0.01 ; 95 \% \mathrm{CI},-0.09$ to $0.06 ; I^{2}=0 \%$ ), or pruritus (RD, $-0.03 ; 95 \% \mathrm{CI},-0.10$ to $0.04 ; I^{2}=0 \%$ ) were reported in the two studies ${ }^{29,38}$ involving 259 patients. Ascites ${ }^{24}$ and fever ${ }^{29}$ were additional indications, as reported in one study each.

\section{Risk of bias}

As previously described in the methodology, the included randomized clinical trial ${ }^{24}$ was assessed for risk of bias using RoB-2. All outcomes analyzed presented an intermediate risk of bias overall, except for the cumulative recurrence-free rate which presented a low risk of bias. The study was hence globally rated as intermediate risk of bias.

Retrospective studies ${ }^{29,36-38}$ were analyzed for risk of bias using ROBINS-I. The outcomes of Kaya et al. ${ }^{29}$ and Han et al ${ }^{38}$ were all at risk of serious bias, giving the studies an overall risk of serious bias. In the study by Navaneethan et al., ${ }^{36}$ the outcomes had a low risk of bias, and an overall low risk of bias was determined. Finally, only the outcomes of Al-Lehibi et al. ${ }^{37}$ presented a risk of critical bias, and was thus globally rated as a critical risk of bias.

The quality of evidence for each of the pre-specified outcomes obtained across the studies was assessed using the GRADE system, as described in the methodology section. Clinical efficacy, recurrence, transplant rate, 5-year survival rate, mortality, pancreatitis, perforation, bleeding, cholangiocarcinoma, in addition to the incidence of jaundice, recurrent cholangitis, abdominal pain, pruritus and fever showed very low quality of evidence. Total complication, the incidence of cholangitis/bacteremia, pain, and ascites also showed low quality of evidence. The only outcome with moderate quality of evidence was cumulative recurrence-free rate, and there were no high quality outcomes.

\section{Results of individual studies}

Ponsioen et al., ${ }^{24}$ the only randomized clinical trial included in this study, concluded that short-term stent was not superior to balloon dilation and associated with higher rates of adverse events, and that balloon dilation should be considered as the initial treatment choice for dominant strictures in PSC patients.

Kaya et al. ${ }^{29}$ also concluded that there were no additional benefits in the use of stents after balloon dilation for the treatment of PSC-related dominant strictures. In addition, stent placement associated with higher rates of complications.

Navaneethan et al. ${ }^{36}$, likewise, concluded that short-term stents after balloon dilation associated with greater risks of adverse events.

Al-Lehibi et al. ${ }^{37}$ was the only study that demonstrated higher success rates with stents compared to balloon dilation, despite the higher rates of adverse events associated.

Han et al. ${ }^{38}$ concluded that patients who received stents presented with more severe diseases, with higher rates of recurrent cholangitis before the procedure. In addition, these patients demonstrated lower 5-year survival rates, as well as higher rates of transplantation and post-procedure cholangitis, which could reflect disease severity. He also concluded that more ERCP procedures were necessary in patients who received stents to achieve the same clinical efficacy as those who underwent balloon dilation.

\section{DISCUSSION}

This is the first systematic review and meta-analysis comparing balloon dilation and stent placement in the treatment of dominant strictures in PSC patients.

PSC is a chronic, progressive disease with limited effective treatments available outside of transplantation. ${ }^{22,25}$ Despite the unclear long-term benefits of endoscopic therapy, multiple techniques for symptom relief in patients affected by PSC have been studied, particularly the endoscopic treatment of dominant strictures. ${ }^{7,25}$ Studies comparing the endoscopic management of PSC-related dominant strictures are necessary, since such procedures carry the potential to alter the survival and prognosis of patients, although the duration of liver transplant-free survival associated with these interventions remains unclear. ${ }^{23}$ Based on the results of this systematic review and meta-analysis of over 460 patients, stent placement for dominant strictures in PSC appeared to associate with higher complication rates without significant differences in efficacy when compared to balloon dilation.

There is currently a variable number of endoscopic treatment modalities for PSC-related dominant strictures, including multiple dilation modalities (balloon or bougie) and stent placement (plastic or metallic). ${ }^{23,25,27}$ Balloon dilations have been preferred as the first choice of treatment, while stent placement has been reserved for cases with inadequate biliary drainage after dilation, cases of long strictures, or where it is believed that dilation will not provide a lasting effect. ${ }^{23}$ Some endoscopists, however, rely upon stent placement as first-line therapy based on their preference and treatment expertise, ${ }^{23}$ since both treatments have been considered effective in resolving symptoms. ${ }^{22}$ Disadvantages of these methods include early 
restenosis in the case of balloon dilation, and the risk of stent occlusion with consequent cholangitis and sepsis in the case of stent placement. ${ }^{24}$ In addition, stenting requires a second intervention, either for replacement or for removal. ${ }^{24}$

In this meta-analysis of five studies, each study concluded varying success rates and outcomes. Kaya et al..$^{29}$ concluded that there were no additional benefits in the use of stents after balloon dilation for the treatment of PSC-related dominant strictures, and demonstrated that stent placement associated with higher rates of complications. Similarly, Navaneethan et al. ${ }^{36}$ found that short-term stent placement after balloon dilation associated with greater risk of adverse events. In the study by Han et al., ${ }^{38}$ patients who received stents associated with lower 5-year survival rates, and higher rates of recurrent cholangitis, transplantation, and post-procedure cholangitis, suggesting that the outcomes of these two interventions may not be comparable. While these studies have consistently shown poorer outcomes with stent placement, the study by Al-Lehibi et al. ${ }^{37}$ demonstrated higher success rates with stents than with balloon dilation, despite the higher rates of associated adverse events. While the aforementioned studies were retrospective in nature, the only randomized trial by Ponsioen et al. ${ }^{24}$ concluded that short-term stent placement was not superior to balloon dilation, and associated with higher rates of adverse events.

Such finding was very much similar to those of our systematic review and meta-analysis, suggesting that balloon dilation should be considered as the initial treatment choice for dominant strictures among PSC patients.

Most studies, ${ }^{24,29,36,38}$ although not randomized clinical trials, have also reported lower rates of complications with balloon dilation compared to stent placement for PSC-related dominant strictures. Kaya et al., ${ }^{29}$ Ponsioen et al., ${ }^{24}$ Navaneethan et al., ${ }^{36}$ and Han et al., ${ }^{38}$ although with different study designs, reached similar conclusions regarding the efficacy of balloon dilation compared to stent placement, as well as the difference in the occurrence of adverse events between both methods.

The question remains regarding the clinical efficacy of these techniques, which was found to be similar in our analysis. Among the included studies, clinical efficacy was reported in two, ${ }^{37,38}$ which involved a total of 259 patients. In the study by Han et al., ${ }^{38}$ clinical efficacy was higher in the stent group, while in that by Al-Lehibi et al., ${ }^{37}$ it was higher in the balloon dilation group, altogether suggesting a similarity in clinical efficacy between stents and balloon dilation. The remaining studies included in this review did not report such outcome.

Recurrence rate, although only reported in one study, ${ }^{29}$ was favored in stent placement rather than balloon dilation. However, no other studies have reported such outcome, rendering comparison impossible.

The same occurred with cumulative recurrence-free rate, with only one study ${ }^{24}$ reporting higher rates in the dilation group, favoring its use instead of stent placement.

Transplant rate, on the other hand, was reported in two studies, ${ }^{29,38}$ both of which demonstrated higher but insignificant rates in the dilation group.

Similarly, the 5-year survival rate was reported in only one study, ${ }^{38}$ and showed higher rates in the stent placement group, favoring its use instead of balloon dilation.

In terms of adverse events, total number of events were reported by three studies, ${ }^{24,29,36}$ all of which showed higher rates in the stent group, thereby favoring the balloon dilation approach. It is worth mentioning that recurrent cholangitis was found to be more common in the balloon dilation cohort than in the stent placement group; this may discount the possibility of stent infection and failure being attributable to the greater number of patients with existing infections.

Standardized data across the included studies would have improved the quality of comparison made in our study; the lack of this in several of the reported outcomes represented a limiting factor of our meta-analysis. All of these factors contributed to the low or very low quality of evidence in the vast majority of the outcomes. The evidence of only one outcome (cumulative recurrence-free rate) was of moderate quality; however, as it was only reported in one study, ${ }^{24}$ a meta-analysis remained unfeasible. Other limitations of the present study included the isolated analysis of a randomized clinical trial, the combined assessment of the randomized clinical trial with the retrospective studies in the meta-analysis, and the lack of uniformity in the evaluated outcomes. In addition, the quality of the evidence generated in the analysis was low, limiting the generalization power of the study.

The lack of randomized clinical trials on the endoscopic management approach of PSC-related dominant strictures demonstrates that this remains a poorly explored topic, despite the clinical potential of balloon dilation and stent placement in increasing survival and improving the quality of life of such patients. Given the scarcity of definitive treatments such as liver transplantation, further investigation of these endoscopic therapies is highly relevant to clinical investigators. Just as studies have improved the diagnostic algorithm and identified a cost-effective approach for indeterminant strictures among patients with PSC, further treatment guidelines and cost-effectiveness analyses are needed for the treatment of dominant strictures. ${ }^{3,39}$

Our study had several limitations, such as the moderate heterogeneity in patient populations and the differences in techniques applied in each study. Furthermore, only three studies ${ }^{24,29,36}$ clarified that both groups (dilation and stent) were comparable at baseline in terms of symptoms, disease duration, and bilirubin level; while the two other studies ${ }^{37,38}$ did not 
include such detail. None of the studies reported the duration or severity of strictures. Further studies with well-designed randomized trials may help determine the optimal endoscopic treatment algorithms for PSC-related dominant strictures, and guide clinical decision making and endoscopic practice patterns.

\section{CONCLUSIONS}

Balloon dilation and stent placement demonstrated similar clinical efficacy in resolving the symptoms of cholestasis and dominant strictures among PSC patients. However, biliary stent placement appeared to increase the risk of total adverse events, specifically that of cholangitis/bacteremia.

\author{
Conflicts of Interest \\ The authors have no potential conflicts of interest. \\ Funding \\ None. \\ Author Contributions \\ Conceptualization: Marina Tucci Gammaro Baldavira Ferreira, Igor Bra- \\ ga Ribeiro, Diogo Turiani Hourneaux de Moura, Thomas R. McCarty, \\ Alberto Machado da Ponte Neto, Galileu Ferreira Ayala Faria, Antônio \\ Afonso de Miranda Neto, Pedro Victor Aniz Gomes de Oliveira, Wan- \\ derley Marques Bernardo, Eduardo Guimarães Hourneaux de Moura \\ Data curation: MTGBF, WMB \\ Formal analysis: DTHM, WMB, EGHM \\ Investigation: MTGBF, IBR \\ Methodology: MTGBF, IBR, EGHM \\ Project administration: MTGBF, WMB, EGHM \\ Resources: EGHM \\ Software: MTGBF, WMB \\ Supervision: DTHM, WMB, EGHM \\ Validation: WMB, EGHM \\ Visualization: TRM, IBR, DTHM, WMB, EGHM \\ Writing-original draft: MTGBF, IBR \\ Writing-review\&editing: TRM, DTHM, WMB, EGHM
}

\section{ORCID}

Marina Tucci Gammaro Baldavira Ferreira: https://orcid.org/0000-0002-7808-5601 Igor Braga Ribeiro: https://orcid.org/0000-0003-1844-8973

Diogo Turiani Hourneaux deMoura: https://orcid.org/0000-0002-7446-0355

Thomas McCarty: https://orcid.org/0000-0003-4517-5261

Alberto Machado da Ponte Neto: https://orcid.org/0000-0001-6652-9159

Galileu Ferreira Ayala Farias: https://orcid.org/0000-0003-0242-3691

Antônio Afonso de Miranda Neto: https://orcid.org/0000-0002-9439-9088

Pedro Victor Aniz Gomes de Oliveira: https://orcid.org/0000-0002-4378-973X

Wanderley Marques Bernardo: https://orcid.org/0000-0002-8597-5207

Eduardo Guimarães Hourneaux de Moura: https://orcid.org/0000-0002-8023-3722

\section{REFERENCES}

1. Lee YM, Kaplan MM. Primary sclerosing cholangitis. N Engl J Med
1995;332:924-933.

2. Angulo P, Lindor KD. Primary sclerosing cholangitis. Hepatology 1999;30:325-332.

3. Njei B, McCarty TR, Varadarajulu S, Navaneethan U. Systematic review with meta-analysis: endoscopic retrograde cholangiopancreatography-based modalities for the diagnosis of cholangiocarcinoma in primary sclerosing cholangitis. Aliment Pharmacol Ther 2016;44:1139-1151.

4. Tabibian JH, Baron TH. Endoscopic management of primary sclerosing cholangitis. Expert Rev Gastroenterol Hepatol 2018;12:693-703.

5. Broomé U, Olsson R, Lööf L, et al. Natural history and prognostic factors in 305 Swedish patients with primary sclerosing cholangitis. Gut 1996;38:610-615.

6. Sclair SN, Little E, Levy C. Current concepts in primary biliary cirrhosis and primary sclerosing cholangitis. Clin Transl Gastroenterol 2015;6:e109.

7. Sedki M, Levy C. Update in the care and management of patients with primary sclerosing cholangitis. Curr Gastroenterol Rep 2018;20:29.

8. Karlsen TH, Folseraas T, Thorburn D, Vesterhus M. Primary sclerosing cholangitis-a comprehensive review. J Hepatol 2017;67:1298-1323.

9. Weismüller TJ, Trivedi PJ, Bergquist A, et al. Patient age, sex, and inflammatory bowel disease phenotype associate with course of primary sclerosing cholangitis. Gastroenterology 2017;152:1975-1984.e8.

10. de Moura DTH, Ryou M, de Moura EGH, Ribeiro IB, Bernardo WM, Thompson CC. Endoscopic ultrasound-guided fine needle aspiration and endoscopic retrograde cholangiopancreatography-based tissue sampling in suspected malignant biliary strictures: a meta-analysis of same-session procedures. Clin Endosc 2020;53:417-428.

11. Abu-Wasel B, Keough V, Renfrew PD, Molinari M. Biliary stent therapy for dominant strictures in patients affected by primary sclerosing cholangitis. Pathobiology 2013;80:182-193.

12. Moura DTH, de Moura EGH, Matuguma SE, et al. EUS-FNA versus ERCP for tissue diagnosis of suspect malignant biliary strictures: a prospective comparative study. Endosc Int Open 2018;6:E769-E777.

13. de Oliveira PVAG, de Moura DTH, Ribeiro IB, et al. Efficacy of digital single-operator cholangioscopy in the visual interpretation of indeterminate biliary strictures: a systematic review and meta-analysis. Surg Endosc 2020;34:3321-3329.

14. Delgado AA de A, de Moura DTH, Ribeiro IB, et al. Propofol vs traditional sedatives for sedation in endoscopy: a systematic review and meta-analysis. World J Gastrointest Endosc 2019;11:573-588.

15. Matsubayashi CO, Ribeiro IB, de Moura DTH, et al. Is endoscopic balloon dilation still associated with higher rates of pancreatitis?: a systematic review and meta-analysis. Pancreas 2020;49:158-174.

16. Funari MP, Ribeiro IB, de Moura DTH, et al. Adverse events after biliary sphincterotomy: does the electric current mode make a difference? a systematic review and meta-analysis of randomized controlled trials. Clin Res Hepatol Gastroenterol 2020;44:739-752.

17. Passos ML, Ribeiro IB, de Moura DTH, et al. Efficacy and safety of carbon dioxide insufflation versus air insufflation during endoscopic retrograde cholangiopancreatography in randomized controlled trials: a systematic review and meta-analysis. Endosc Int Open 2019;7:E487-E497.

18. Serrano JPR, de Moura DTH, Bernardo WM, et al. Nonsteroidal anti-inflammatory drugs versus placebo for post-endoscopic retrograde cholangiopancreatography pancreatitis: a systematic review and meta-analysis. Endosc Int Open 2019;7:E477-E486.

19. European Society of Gastrointestinal Endoscopy, European Association for the Study of the Liver. Role of endoscopy in primary sclerosing cholangitis: european society of gastrointestinal endoscopy (ESGE) and european association for the study of the liver (EASL) clinical guideline. J Hepatol 2017;66:1265-1281.

20. Chapman MH, Thorburn D, Hirschfield GM, et al. British society of gastroenterology and UK-PSC guidelines for the diagnosis and management of primary sclerosing cholangitis. Gut 2019;68:1356-1378.

21. Logiudice FP, Bernardo WM, Galetti F, et al. Endoscopic ultrasound-guid- 
ed vs endoscopic retrograde cholangiopancreatography biliary drainage for obstructed distal malignant biliary strictures: a systematic review and meta-analysis. World J Gastrointest Endosc 2019;11:281-291.

22. Parsi MA. Common controversies in management of biliary strictures. World J Gastroenterol 2017;23:1119-1124.

23. Modha K, Navaneethan U. Diagnosis and management of primary sclerosing cholangitis-perspectives from a therapeutic endoscopist. World J Hepatol 2015;7:799-805.

24. Ponsioen CY, Arnelo U, Bergquist A, et al. No superiority of stents vs balloon dilatation for dominant strictures in patients with primary sclerosing cholangitis. Gastroenterology 2018;155:752-759.e5.

25. Costamagna G, Boškoski I. Current treatment of benign biliary strictures. Ann Gastroenterol 2013;26:37-40.

26. De Moura DTH, Moura EGHD, Bernardo WM, et al. Endoscopic retrograde cholangiopancreatography versus endoscopic ultrasound for tissue diagnosis of malignant biliary stricture: systematic review and meta-analysis. Endosc Ultrasound 2018;7:10-19.

27. Visconti TA de C, Bernardo WM, Moura DTH, et al. Metallic vs plastic stents to treat biliary stricture after liver transplantation: a systematic review and meta-analysis based on randomized trials. Endosc Int Open 2018;6:E914-E923.

28. Ferreira R, Loureiro R, Nunes N, et al. Role of endoscopic retrograde cholangiopancreatography in the management of benign biliary strictures: what's new? World J Gastrointest Endosc 2016;8:220-231.

29. Kaya M, Petersen BT, Angulo P, et al. Balloon dilation compared to stenting of dominant strictures in primary sclerosing cholangitis. Am J Gastroenterol 2001;96:1059-1066.

30. Baron TH, Davee T. Endoscopic management of benign bile duct strictures. Gastrointest Endosc Clin N Am 2013;23:295-311.

31. Moher D, Shamseer L, Clarke M, et al. Preferred reporting items for systematic review and meta-analysis protocols (PRISMA-P) 2015 statement. Syst Rev 2015;4:1.

32. Sterne JAC, Savović J, Page MJ, et al. RoB 2: a revised tool for assessing risk of bias in randomised trials. BMJ 2019;366:14898.

33. Sterne JA, Hernán MA, Reeves BC, et al. ROBINS-I: a tool for assessing risk of bias in non-randomised studies of interventions. BMJ 2016;355:14919.

34. Morgan RL, Florez I, Falavigna M, et al. Development of rapid guidelines: 3. GIN-Mcmaster guideline development checklist extension for rapid recommendations. Health Res Policy Syst 2018;16:63.

35. Hozo SP, Djulbegovic B, Hozo I. Estimating the mean and variance from the median, range, and the size of a sample. BMC Med Res Methodol 2005;5:13.

36. Navaneethan U, Jegadeesan R, Hammel J, Sanaka MR, Vargo JJ, Parsi MA. Su1646 endoscopic treatment of dominant biliary strictures in primary sclerosing cholangitis patients: comparison of adverse events associated with balloon dilation with and without stenting. Gastrointestinal Endoscopy 2015;81:AB364.

37. Al-Lehibi AH, Kumar N, Ammar T, et al. T1572: experience with endoscopic therapy in patients with primary sclerosing cholangitis. Gastrointestinal Endoscopy 2010;71:AB312.

38. Han S, Xu A, Shah RJ. Tu1435 ERCP with balloon dilation alone or balloon dilation with stenting for dominant strictures in patients with primary sclerosing cholangitis. Gastrointestinal Endoscopy 2017;85:AB625AB626.

39. Njei B, McCarty TR, Varadarajulu S, Navaneethan U. Cost utility of ERCP-based modalities for the diagnosis of cholangiocarcinoma in primary sclerosing cholangitis. Gastrointestinal Endoscopy 2017;85:773781.e10. 